EPJ Web of Conferences 60, 16006 (2013)

DOI: $10.1051 /$ epjconf $/ 20136016006$

(C) Owned by the authors, published by EDP Sciences, 2013

\title{
Tevatron combined top quark mass
}

\author{
P. Bartoš ${ }^{1, a}$ on behalf of CDF and D $\varnothing$ Collaborations \\ ${ }^{1}$ Faculty of Mathematics, Physics and Inforamtics, Comenius University - Mlynska Dolina F1 Bratislava 842 48, Slovakia
}

\begin{abstract}
We summarize the top-quark mass measurements from the CDF and D0 experiments at Fermilab. We combine published Run I (1992-1996) measurements with the most precise published and preliminary Run II (2001-2012) measurements using a data set corresponding to up to $8.7 \mathrm{fb}^{-1}$ of $p \bar{p}$ collisions. Taking uncertainty correlations into account, and adding in quadrature the statistical and systematic uncertainties, the resulting preliminary Tevatron average mass of the top quark is $m_{t}=173.20 \pm 0.87 \mathrm{GeV} / c^{2}$.
\end{abstract}

\section{Introduction}

The top quark is the heaviest fundamental particle with the unique properties. It is $3^{\text {rd }}$ generation quark with electric charge of $+2 / 3 e$ and mass of $173.20 \pm 0.87 \mathrm{GeV} / c^{2}$ [1]. The huge mass gives importance to the QCD corrections for the top quark. Due to the very short lifetime $\left(\sim 10^{-25} \mathrm{~s}\right)$, the top quark decays before hadronization and we can study its properties using its decay products. If we see a deviation of the measured properties from the Standard Model predictions, it could be a sign of new physics. The Yukawa coupling of the top quark is close to unity, what raises the question if it has a special role in the electroweak symmetry breaking. In addition to that the topquark events are important background in the Higgs-boson studies.

At the Tevatron $p \bar{p}$ collider, top quarks are produced mainly in pairs through strong force quark-antiquark annihilation $(\sim 85 \%)$ and gluon-gluon fusion $(\sim 15 \%)$ processes. As the top quark decays into the $W$ boson and bottom $(b)$ quark in almost $100 \%$ of the cases, the final state of top-quark-pair production contains two b-quarks jets and two $W$ bosons, which decay leptonically (to $l v_{l}$, where in our case $l=e, \mu$ ) or hadronically (into quarks). The $t \bar{t}$ events can be then classified into three categories : the dilepton or all-jets events, where both $W$ bosons decay leptonically or hadronically, respectively; and the lepton+jets events, where one of the $W$ bosons decays leptonically while the other one decays hadronically.

In this article we present the combination of the 12 different top-quark-mass measurements done by the CDF and $\mathrm{D} \varnothing$ collaborations using a data set corresponding to up to $8.7 \mathrm{fb}^{-1}$.

\section{Input measurements}

The twelve measurements used in this combination are summarized in Tab. 1.

\footnotetext{
a. e-mail: bartos.palik@gmail.com
}

\subsection{Run I}

The first measurements used Run I data (collected from 1992 to 1996) of integrated luminosity of $0.1 \mathrm{fb}^{-1}$. There are tree measurements done by CDF [2-5] and two done by $\mathrm{D} \varnothing[6,7]$ collaboration - all have relatively large statistical uncertainties. Their systematic uncertainties are dominated by the total jet energy scale (JES) uncertainty.

\subsection{Run II}

The measurements, which use Run II data (collected from 2001-2011), are the most recent results in several different decay channels and use up to $8.7 \mathrm{fb}^{-1}$ of data. The statistical and systematic uncertainties are reduced by studying much larger $t \bar{t}$ samples and using new analysis techniques.

\subsubsection{CDF measurements}

The lepton+jets analysis is published result [8], which uses full statistics of collected data $\left(8.7 \mathrm{fb}^{-1}\right)$. We constrain the response of light-quark jets using the kinematic information from $W \rightarrow q q^{\prime}$ decays (in situ calibration) to reduce JES uncertainty. Residual JES uncertainties associated with transverse momenta or pseudo-rapidity dependencies and uncertainties specific to the b-jets response are treated separately. We improved jet energy resolution with respect to the previous combination [9].

The dilepton [10] and all-jets [11] analyses use sample of $5.6 \mathrm{fb}^{-1}$ and $5.8 \mathrm{fb}^{-1}$, respectively, and are unchanged with respect to previous combination [9].

The missing-transverse-energy (MEt) analysis [12] shows preliminary result updated with $8.7 \mathrm{fb}^{-1}$ of data (full data set). In this analysis, events are required to have a missing transverse energy, jets and none identified lepton. The sample is statistically independent from above mentioned three categories and is considered as fourth. The JES uncertainty is also determined by in situ calibration. 
The analysis based on charged-particle tracking uses $1.9 \mathrm{fb}^{-1}$ of data [13]. This technique uses the decay length of B-mesons from $b$-jets $L_{X Y}$. This analysis is almost entirely independent of JES uncertainties, but the statistical sensitivity is not so good as for previously mentioned measurements.

\subsubsection{DØ measurements}

The two measurements used in this combination include most recent results and are both published.

The lepton+jets measurement [14], based on $3.6 \mathrm{fb}^{-1}$ of data, uses JES determined from the external calibration derived from $\gamma+$ jets events as an additional Gaussian constrain to the in situ calibration.

The dilepton analysis [15] uses $5.4 \mathrm{fb}^{-1}$ of data. In this case we use JES determined in the lepton+jet measurement by in situ calibration.

\section{Uncertainty Categories}

The uncertainties of the measurements are divided into several categories (see [9] for details). The categories are set in such a way, that uncertainties with the same or similar origin are combined (i. e., Signal category below), while some uncertainties have been separated into multiple categories in order to accommodate specific types of correlations (JES uncertainties below).

In situ light-jet calibration - originates from in situ calibration procedures and is uncorrelated among the measurements. It is part of the JES uncertainty.

Response to $b / q / g$ jets - comes from differences in the detectors response to $b$-jets and light-quark jets. It is part of the JES uncertainty.

Model for $b$-jets - uncertainty, which originates from the $b$-jet modeling. It includes uncertainties coming from variations in the semileptonic branching fractions, bfragmentation modeling, and differences in the color flow between $b$-jets and light-quark jets. It is part of the JES uncertainty.

Out-of-cone correction - this part of JES uncertainty is correlated across all measurements. It includes the uncertainties originated in modeling of light-quark fragmentation and out-of-cone corrections.

Light-jet response (1) - is specific to the CDF. It includes uncertainties associated with calorimeter response to light-quark jets, multiple interaction and underlying events corrections. This uncertainty is correlated across all CDF measurements independently from the data-taking period (i.e., Run I or Run II). It is uncorrelated between experiments. It is part of the JES uncertainty.

Light-jet response (2) - is the uncertainty coming from limitation in the data samples used for calibrations. It is correlated between measurements from the same datataking period. It is not correlated between experiments. For the $\mathrm{CDF}$, it corresponds to uncertainties associated with the $\eta$-dependent JES corrections. For the D $\varnothing$ it includes uncertainties coming from calorimeter response to light jets, uncertainties from $p_{T}$ - and $\eta$-dependent JES corrections anad from the sample dependence of using $\gamma+$ jets data samples to derive the JES.

Lepton modeling - originates from the uncertainties in the scale of the lepton $p_{T}$ measurements. It is not treated as a source of systematic uncertainty in the Run I measurements.

Signal modeling - is uncertainty arising from $t \bar{t}$ modeling and is correlated across all measurements. In includes uncertainties from variation of the amount of initial and final state radiation and from the choice of parton density function. For D $\varnothing$ it also includes uncertainty from higher order correction (NLO). It also include the uncertainty arising from a variation of color reconnection model (this uncertainty was not evaluated in Run I measurements). Finally, it takes into account the uncertainty coming from the choice of Monte Carlo generator, which is used to $t \bar{t}$ signal modeling.

Jet modeling - arises from uncertainties in the detector modeling in the MC simulation. The $\mathrm{D} \varnothing$ includes also uncertainties from jet resolution and identification, while these effects where found to be negligible for mass measurements at the CDF.

Background from theory - takes care of uncertainty in modeling the background sources. It includes uncertainties of background composition, normalization and shape of different components. It is correlated across all measurements in the same channel.

Background based on data - takes into account uncertainties arising from modeling QCD multijets (in lepton+jets, all-hadonic, and MEt channel) or Drell-Yan (in dilepton channel) background using data. It is uncorrelated between experiments.

Calibration method - comes from any source specific to a particular fit method. It also includes uncertainty arising from using finite MC statistics to the calibration of each method.

Offset - is specific to the D $\varnothing$. It comes from uranium noise in the calorimeter and from the multiple interaction correction to the JES. For Run I it was sizable, while for Run II measurements it is negligible.

Multiple interaction model - originates in mismodeling of the distribution of the number of collisions per Tevatron bunch crossing. It is uncorrelated between experiments.

All above mentioned systematic uncertainties for each of input measurements are summarize in Tab. 1.

\section{Combination}

The measurements are combined by two independent methods - numerical $\chi^{2}$ minimization and the analytic best linear unbiased estimator (BLUE) method $[16,17]$. These two methods are mathematically equivalent and give identical results for the combination. The BLUE method yields the decomposition of the uncertainty on the Tevatron combined top-quark mass $M_{t}$ in terms of the uncertainty categories specified for the input measurements [17].

For the combination we used following correlations : 
Table 1. Summary of the measurements used to determine the combined top-quark mass. Integrated luminosity has units of $\mathrm{fb}^{-1}$, other numbers are in $\mathrm{GeV} / c^{2}$. Term "n/a" ("n/e") stands for "not applicable" ("not evaluated"). The total systematic uncertainty and the total uncertainty are obtained by adding the relevant contributions in quadrature.

\begin{tabular}{|c|c|c|c|c|c|c|c|c|c|c|c|c|}
\hline & \multicolumn{11}{|c|}{ March 2013} & \multirow{4}{*}{$\begin{array}{c}\text { Run II preliminary } \\
\text { CDF } \\
\text { MEt }\end{array}$} \\
\hline & \multicolumn{5}{|c|}{ Run I published } & \multicolumn{6}{|c|}{ Run II published } & \\
\hline & \multicolumn{3}{|c|}{$\mathrm{CDF}$} & \multicolumn{2}{|c|}{$\mathrm{D} \varnothing$} & \multicolumn{4}{|c|}{ CDF } & \multicolumn{2}{|c|}{$\mathrm{D} \varnothing$} & \\
\hline & $l+$ jets & $l l$ & alljets & $l+$ jets & $l l$ & $l+$ jets & $l l$ & alljets & $L_{X Y}$ & $l+$ jets & $l l$ & \\
\hline Luminosity & 0.1 & 0.1 & 0.1 & 0.1 & 0.1 & 8.7 & 5.6 & 5.8 & 1.9 & 3.6 & 5.3 & 8.7 \\
\hline Result & 176.1 & 167.4 & 86.0 & 180.1 & 168.4 & 172.85 & 170.28 & 172.47 & 166.90 & 174.94 & 174.00 & 173.95 \\
\hline $\begin{array}{l}\text { In situ light-jet } \\
\text { calibration }\end{array}$ & $\mathrm{n} / \mathrm{a}$ & $\mathrm{n} / \mathrm{a}$ & $\mathrm{n} / \mathrm{a}$ & $\mathrm{n} / \mathrm{a}$ & $\mathrm{n} / \mathrm{a}$ & 0.49 & $\mathrm{n} / \mathrm{a}$ & 0.95 & $\mathrm{n} / \mathrm{a}$ & 0.53 & 0.55 & 1.05 \\
\hline $\begin{array}{l}\text { Response to } b / q / g \\
\text { jets }\end{array}$ & $\mathrm{n} / \mathrm{a}$ & $\mathrm{n} / \mathrm{a}$ & $\mathrm{n} / \mathrm{a}$ & 0.0 & 0.0 & 0.09 & 0.14 & 0.03 & $\mathrm{n} / \mathrm{a}$ & 0.0 & 0.40 & 0.10 \\
\hline Model for $b$ jets & 0.6 & 0.8 & 0.6 & 0.7 & 0.7 & 0.16 & 0.33 & 0.15 & $\mathrm{n} / \mathrm{a}$ & 0.07 & 0.20 & 0.17 \\
\hline $\begin{array}{l}\text { Out-of-cone correc- } \\
\text { tion }\end{array}$ & 2.7 & 2.6 & 3.0 & 2.0 & 2.0 & 0.21 & 2.13 & 0.24 & 0.36 & $\mathrm{n} / \mathrm{a}$ & $\mathrm{n} / \mathrm{a}$ & 0.18 \\
\hline $\begin{array}{c}\text { Light-jet respon- } \\
\text { se (2) }\end{array}$ & 0.7 & 0.6 & 0.3 & 2.5 & 1.1 & 0.07 & 0.58 & 0.04 & 0.06 & 0.63 & 0.56 & 0.04 \\
\hline $\begin{array}{l}\text { Light-jet respon- } \\
\text { se (1) }\end{array}$ & 3.4 & 2.7 & 4.0 & $\mathrm{n} / \mathrm{a}$ & $\mathrm{n} / \mathrm{a}$ & 0.48 & 2.01 & 0.38 & 0.24 & $\mathrm{n} / \mathrm{a}$ & $\mathrm{n} / \mathrm{a}$ & 0.40 \\
\hline Lepton modeling & $\mathrm{n} / \mathrm{e}$ & $\mathrm{n} / \mathrm{e}$ & $\mathrm{n} / \mathrm{e}$ & $\mathrm{n} / \mathrm{e}$ & $\mathrm{n} / \mathrm{e}$ & 0.03 & 0.27 & $\mathrm{n} / \mathrm{a}$ & $\mathrm{n} / \mathrm{a}$ & 0.17 & 0.35 & $\mathrm{n} / \mathrm{a}$ \\
\hline Signal modeling & 2.6 & 2.9 & 2.0 & 1.1 & 1.8 & 0.61 & 0.73 & 0.62 & 0.90 & 0.77 & 0.86 & 0.64 \\
\hline Jet modeling & 0.0 & 0.0 & 0.0 & 0.0 & 0.0 & 0.0 & 0.0 & 0.0 & 0.0 & 0.36 & 0.50 & 0.0 \\
\hline Offset & $\mathrm{n} / \mathrm{a}$ & $\mathrm{n} / \mathrm{a}$ & $\mathrm{n} / \mathrm{a}$ & 1.3 & 1.3 & $\mathrm{n} / \mathrm{a}$ & $\mathrm{n} / \mathrm{a}$ & $\mathrm{n} / \mathrm{a}$ & $\mathrm{n} / \mathrm{a}$ & $\mathrm{n} / \mathrm{a}$ & $\mathrm{n} / \mathrm{a}$ & $\mathrm{n} / \mathrm{a}$ \\
\hline $\begin{array}{l}\text { Background from } \\
\text { theory }\end{array}$ & 1.3 & 0.3 & 1.7 & 1.0 & 1.1 & 0.12 & 0.24 & 0.0 & 0.80 & 0.18 & 0.0 & 0.0 \\
\hline $\begin{array}{l}\text { Background based } \\
\text { on data }\end{array}$ & 0.0 & 0.0 & 0.0 & 0.0 & 0.0 & 0.16 & 0.14 & 0.56 & 0.20 & 0.23 & 0.20 & 0.12 \\
\hline Calibration method & 0.0 & 0.7 & 0.6 & 0.6 & 1.1 & 0.00 & 0.12 & 0.38 & 2.50 & 0.16 & 0.51 & 0.31 \\
\hline $\begin{array}{l}\text { Multiple interac- } \\
\text { tions model }\end{array}$ & $\mathrm{n} / \mathrm{e}$ & $\mathrm{n} / \mathrm{e}$ & $\mathrm{n} / \mathrm{e}$ & $\mathrm{n} / \mathrm{e}$ & $\mathrm{n} / \mathrm{e}$ & 0.07 & 0.23 & 0.08 & 0.0 & 0.05 & 0.0 & 0.18 \\
\hline $\begin{array}{l}\text { Systematic } \\
\text { uncertainty }\end{array}$ & 5.3 & 4.9 & 5.7 & 3.9 & 3.6 & 0.98 & 3.09 & 1.49 & 2.90 & 1.24 & 1.44 & 1.35 \\
\hline $\begin{array}{l}\text { Statistical } \\
\text { uncertainty }\end{array}$ & 5.1 & 10.3 & 10.0 & 3.6 & 12.3 & 0.52 & 1.95 & 1.43 & 9.00 & 0.83 & 2.36 & 1.26 \\
\hline Total uncertainty & 7.3 & 11.4 & 11.5 & 5.3 & 12.8 & 1.11 & 3.79 & 2.06 & 9.46 & 1.50 & 2.76 & 1.85 \\
\hline
\end{tabular}

- The Statistical uncertainty, Calibration method, and In situ light-jet calibration uncertainties are treated as uncorrelated across the measurements.

- The uncertainties in the Response to b/q/g jets, Lightjet response (2), Lepton modeling, and Multiple interaction model categories are taken to be $100 \%$ correlated across all measurements within Run I or Run II. They are threated as uncorrelated between Run I and Run II, and also uncorrelated between the experiments.

- The uncertainties in the Light-jet response (1), Jet modeling, and Offset categories are taken to be $100 \%$ correlated among all measurements within the same experiment. They are threated as uncorrelated between the experiments.

- The Backgrounds estimated from theory uncertainties are taken to be $100 \%$ correlated across all measurements in the same decay channel.

- The Backgrounds estimated from data uncertainties are taken to be $100 \%$ correlated among all measurements in the same decay channel and same data-taking period.
They are threated as uncorrelated between the experiments.

- The uncertainties in the Model for b-jets, Out-of-cone correction, and Signal modeling categories are taken to be $100 \%$ correlated across all measurements.

By using the inputs measurements and the above mentioned correlations, we obtain the matrix of total correlation coefficients, which is shown in Table 2. The pull and weight for each of the inputs measurements are listed in Table 3. The weights of some measurements are negative, what means that the correlation between two measurements is larger than the ratio of their total uncertainties. In such a case the less precise measurement is assigned by a negative weight. Weight of zero would mean that a input measurement is ignored in the combination. Negative weight means that it affects the resulting combined value and helps reduce the total uncertainty.

We show the absolute values of the weight of each measurement divided by the sum of the absolute values of the weights of all input measurements in Figure 1 to give a better picture of the weights. 
Table 2. The matrix of correlation coefficients used to determine the Tevatron combined top-quark mass.

\begin{tabular}{|c|c|c|c|c|c|c|c|c|c|c|c|c|}
\hline \multicolumn{13}{|c|}{ March 2013} \\
\hline & \multicolumn{5}{|c|}{ Run I published } & \multicolumn{6}{|c|}{ Run II published } & \multirow{3}{*}{$\begin{array}{c}\text { Run II preliminary } \\
\text { CDF } \\
\mathrm{MEt}\end{array}$} \\
\hline & \multicolumn{3}{|c|}{$\mathrm{CDF}$} & \multicolumn{2}{|c|}{$\mathrm{D} \emptyset$} & \multicolumn{4}{|c|}{$\mathrm{CDF}$} & \multicolumn{2}{|c|}{$\mathrm{D} \varnothing$} & \\
\hline & $l+$ jets & $l l$ & alljets & $l+$ jets & $l l$ & $l+$ jets & $l l$ & alljets & $L_{X Y}$ & $l+$ jets & $l l$ & \\
\hline CDF Run I, $l+$ jets & 1.00 & 0.29 & 0.32 & 0.26 & 0.11 & 0.49 & 0.54 & 0.25 & 0.07 & 0.21 & 0.12 & 0.27 \\
\hline CDF Run I, $l l$ & 0.29 & 1.00 & 0.19 & 0.15 & 0.08 & 0.29 & 0.32 & 0.15 & 0.04 & 0.13 & 0.08 & 0.17 \\
\hline CDF Run I, all-hadronic & 0.32 & 0.19 & 1.00 & 0.14 & 0.07 & 0.30 & 0.38 & 0.15 & 0.04 & 0.09 & 0.06 & 0.16 \\
\hline DØ Run $\mathrm{I}, l+$ jets & 0.26 & 0.15 & 0.14 & 1.00 & 0.16 & 0.22 & 0.27 & 0.12 & 0.05 & 0.14 & 0.07 & 0.12 \\
\hline DØ Run I, $l l$ & 0.11 & 0.08 & 0.07 & 0.16 & 1.00 & 0.11 & 0.13 & 0.07 & 0.02 & 0.07 & 0.05 & 0.07 \\
\hline CDF Run II, $l+$ jets & 0.49 & 0.29 & 0.30 & 0.22 & 0.11 & 1.00 & 0.48 & 0.29 & 0.08 & 0.30 & 0.18 & 0.33 \\
\hline CDF Run II, $l l$ & 0.54 & 0.32 & 0.38 & 0.27 & 0.13 & 0.48 & 1.00 & 0.25 & 0.06 & 0.11 & 0.07 & 0.26 \\
\hline CDF Run II, all-jets & 0.25 & 0.15 & 0.15 & 0.12 & 0.07 & 0.29 & 0.25 & 1.00 & 0.04 & 0.16 & 0.10 & 0.17 \\
\hline CDF Run II, $L_{X Y}$ & 0.07 & 0.04 & 0.04 & 0.05 & 0.02 & 0.08 & 0.06 & 0.04 & 1.00 & 0.06 & 0.03 & 0.04 \\
\hline DØ Run II, $l+$ jets & 0.21 & 0.13 & 0.09 & 0.14 & 0.07 & 0.30 & 0.11 & 0.16 & 0.06 & 1.00 & 0.39 & 0.18 \\
\hline DØ Run II, $l l$ & 0.12 & 0.08 & 0.06 & 0.07 & 0.05 & 0.18 & 0.07 & 0.10 & 0.03 & 0.39 & 1.00 & 0.11 \\
\hline CDF Run II, MEt & 0.27 & 0.17 & 0.16 & 0.12 & 0.07 & 0.33 & 0.26 & 0.17 & 0.04 & 0.18 & 0.11 & 1.00 \\
\hline
\end{tabular}

Table 3. The pull and weight for each of the input measurements used to determine the Tevatron combined top-quark mass.

\begin{tabular}{|c|c|c|c|c|c|c|c|c|c|c|c|c|}
\hline \multicolumn{13}{|c|}{ March 2013} \\
\hline & \multicolumn{5}{|c|}{ Run I published } & \multicolumn{6}{|c|}{ Run II published } & \multirow{3}{*}{$\begin{array}{c}\text { Run II preliminary } \\
\text { CDF } \\
\text { MEt }\end{array}$} \\
\hline & \multicolumn{3}{|c|}{$\mathrm{CDF}$} & \multicolumn{2}{|c|}{$\mathrm{D} \varnothing$} & \multicolumn{4}{|c|}{$\mathrm{CDF}$} & \multicolumn{2}{|c|}{$\mathrm{D} \emptyset$} & \\
\hline & $l+$ jets & $l l$ & alljets & $l+$ jets & $l l$ & $l+$ jets & $l l$ & alljets & $L_{X Y}$ & $l+$ jets & $l l$ & \\
\hline Pull & +0.40 & -0.51 & +1.11 & +1.32 & -0.38 & -0.51 & -0.82 & -0.41 & -0.67 & +1.42 & +0.30 & +0.45 \\
\hline Weight [\%] & -4.7 & -1.1 & -0.9 & +0.4 & -0.2 & +62.0 & -0.3 & +10.5 & +0.22 & +20.6 & +1.4 & +11.9 \\
\hline
\end{tabular}

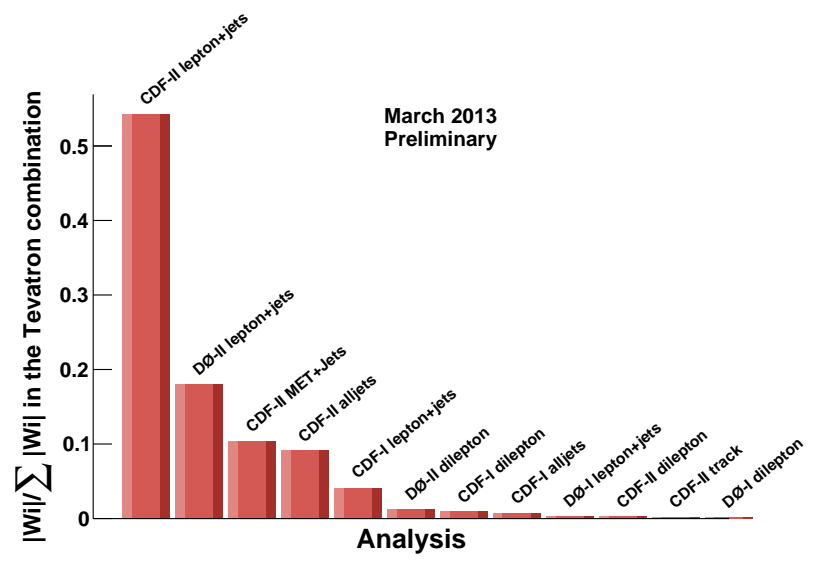

Figure 1. Relative weights of the input measurements in the combination.

\section{Results}

The value of the combined top-quark mas is $173.20 \pm$ 0.51 (stat) \pm 0.71 (syst) $\mathrm{GeV} / c^{2}$. The total uncertainty obtained by adding the statistical and systematic uncertainties in quadrature is $0.87 \mathrm{GeV} / c^{2}$, what corresponds to a relative precision of $0.50 \%$. It has a $\chi^{2}$ of 8.5 for 11 degrees of freedom, what corresponds to a probability of $67 \%$. It indicates good agreement among all input measurements. No input has an anomalously large pull (see Table 3).

The total statistical and systematic uncertainties are slightly smaller with respect to the last published combination [9] due to the using full data set in CDF lepton+jets and MEt measurements and due to the improvements in the treatment of JES corrections. The inputs measurements and the final combined top-quark mass are shown in Fig. 2 .

\subsection{Cross-checks}

As a cross-check, we use the same methodology (inputs, uncertainty categories, and correlations) to determine the top-quark mass in the all-jets $\left(M_{t}^{\text {all-jets }}\right), l+$ jets $\left(M_{t}^{l+j e t s}\right), l l\left(M_{t}^{l l}\right)$, and MEt $\left(M_{t}^{M E t}\right)$ channels, separately. The results of these combinations are shown in Table 4. After expressing a $c h i^{2}$ probabilities, they indicate that all decay channels are consistent with one other.

Table 4. Summary of the calculation of the mass of the top quark in four different decay channels.

\begin{tabular}{cccccc}
\multicolumn{5}{c}{ March 2013 } \\
\hline \hline \multirow{2}{*}{ Parameter } & Value $\left(\mathrm{GeV} / c^{2}\right)$ & $M_{t}^{\text {all-jets }}$ & $M_{t}^{l+\text { jets }}$ & $M_{t}^{l l}$ & $M_{t}^{M E t}$ \\
\hline$M_{t}^{\text {all-jets }}$ & $172.7 \pm 1.9$ & 1.00 & & & \\
$M_{t}^{l+j e t s}$ & $173.2 \pm 0.9$ & 0.25 & 1.00 & & \\
$M_{t}^{l l}$ & $170.0 \pm 2.1$ & 0.19 & 0.41 & 1.00 & \\
$M_{t}^{M E t}$ & $173.8 \pm 1.8$ & 0.13 & 0.26 & 0.18 & 1.00 \\
\hline \hline
\end{tabular}

In order to check how the choice of the correlation affect our result, we do a cross-check by changing all nondiagonal correlation coefficients of the correlation matrix (Table 2) $100 \%$ to $50 \%$ and re-calculate the combination. The result from this test is a $0.19 \mathrm{GeV} / c^{2}$ shift of the topquark mass and a $0.03 \mathrm{GeV} / c^{2}$ decrease of the total uncertainty. 
Mass of the Top Quark

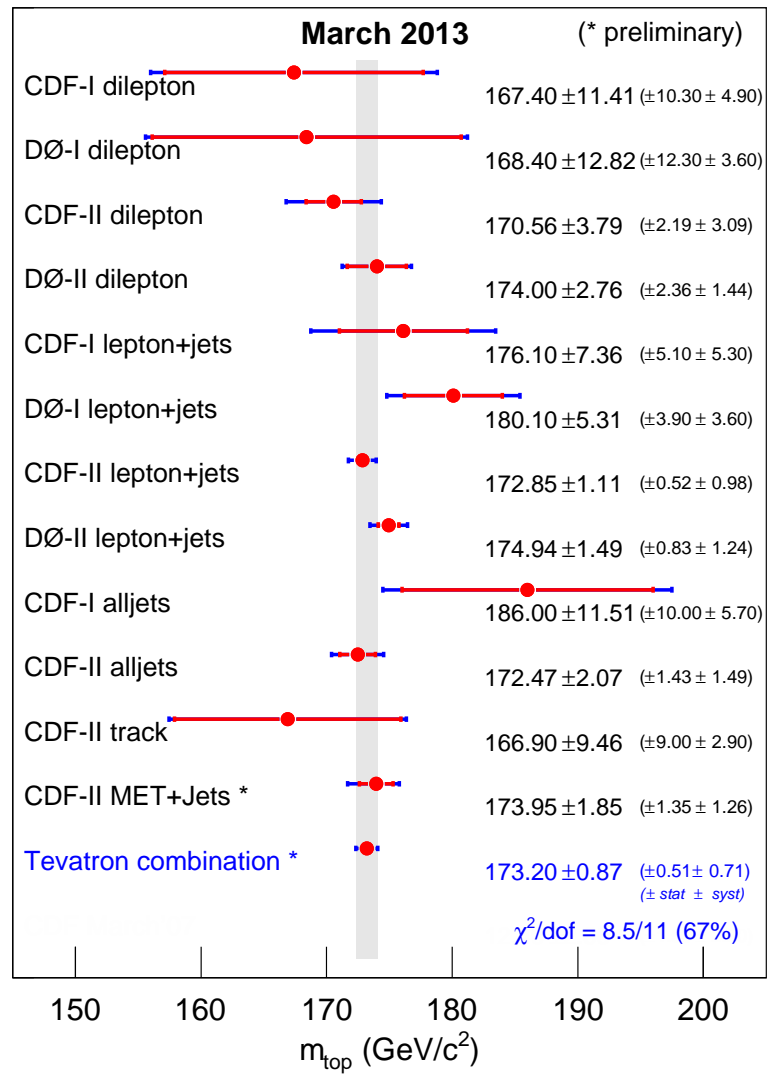

Figure 2. Overview of the top-quark-mass measurements at the Tevatron and the result of their combination.

We also express two separate combinations of all the $\mathrm{CDF}$ and all the $\mathrm{D} \emptyset$ measurements, separately. The results of these combinations are $172.72 \pm 0.93 \mathrm{GeV} / c^{2}$ for $\mathrm{CDF}$ and $174.89 \pm 1.42 \mathrm{GeV} / \mathrm{c}^{2}$ for $\mathrm{D} \emptyset$ measurements. We also calculate the $\chi^{2}(\mathrm{CDF}-\mathrm{D} \emptyset)=2.25 / 1$ corresponding to a probability of $13 \%$.

\section{Conclusion}

A preliminary combination of top-quark mass measurements from the CDF and $\mathrm{D} \varnothing$ experiments is presented. The combination is based on the five published Run I measurements, six published Run II measurements and one preliminary Run II measurement. Taking into account the statistical and systematic uncertainties and their correlations, the preliminary combined results is $M_{t}=$ $173.20 \pm 0.51$ (stat) \pm 0.71 (syst) $\mathrm{GeV} c^{2}$ or $M_{t}=173.20 \pm$
$0.87 \mathrm{GeV} c^{2}$, what corresponds to a relative precision of $0.50 \%$ on the top-quark mass. The result is limited by the systematic uncertainties, which are dominated by the jet energy scale uncertainty.

\section{Acknowledgments}

It is a pleasure to thank the CDF and $\mathrm{D} \emptyset$ collaborators for their well-done work, the top-group conveners for their help and the organizers of the LHCP 2013 for a very interesting conference.

\section{References}

[1] Tevatron EW Working Group, CDF and DØ Collaborations, arXiv :1305.3929 [hep-ex]

[2] F. Abe et al. (CDF Collaboration), Phys. Rev. Lett. 82 271 (1999), arXiv :9810029 [hep-ex]

[3] F. Abe et al. (CDF Collaboration), Phys. Rev. Lett. 82 2808 (1999), arXiv :9810029 [hep-ex]

[4] T. Affolder et al. (CDF Collaboration), Phys. Rev. D 63032003 (2001), arXiv :0006028 [hep-ex]

[5] F. Abe et al. (CDF Collaboration), Phys. Rev. Lett. 79 1992 (1997)

[6] B. Abbott et al. (DØ Collaboration), Phys. Rev. D 60 052001 (1999), arXiv :9808029 [hep-ex]

[7] V. M. Abazov et al. (DØ Collaboration), Nature 429 638 (2004), arXiv :0406031 [hep-ex]

[8] T. Aaltonen et al. (CDF Collaboration), Phys. Rev. Lett. 109152003 (2012)

[9] T. Aaltonen et al. (CDF and DØ Collaborations), Phys. Rev. D 86092003 (2012)

[10] T. Aaltonen et al. (CDF Collaboration), Phys. Rev. D 81032002 (2010), Phys. Rev. D 83111101 (2011), arXiv : 1105.0192 [hep-ex]

[11] T. Aaltonen et al. (CDF Collaboration), Phys. Lett. B 714, 24 (2012)

[12] T. Aaltonen et al. (CDF Collaboration), CDF Conference Note 10433

[13] T. Aaltonen et al. (CDF Collaboration), Phys. Rev. D 81032002 (2010)

[14] V .M. Abazov et al. (DØ Collaboration), Phys. Rev. D 84032004 (2011)

[15] V. M. Abazov et al. (DØ Collaboration), Phys. Rev. D 86051103 (2012)

[16] L. Lyons, D. Gibaut, and P. Clifford, Nucl. Instrum. Meth. A270 110 (1988)

[17] A. Valassi, Nucl. Instrum. Meth. A500 391 (2003) 